\title{
Beliefs About Health and Illness in Latin-American Migrants with Diabetes Living in Sweden
}

\author{
Katarina Hjelm* and Karin Bard
}

School of Health and Caring Sciences, Linnaeus University, Växjö, S-35195 Växjö, Sweden

\begin{abstract}
The study explored beliefs about health and illness in Latin American migrants diagnosed with diabetes mellitus (DM) living in Sweden, and investigated the influence on health-related behavior including self-care and careseeking behavior. Migrants are particularly affected in the diabetes pandemia. Beliefs about health and illness determine health-related behaviour and health but no studies have been found on Latin American migrants with DM. An explorative study design with focus-group interviews of nine persons aged 36-77 years from a diabetes clinic was used. Health was described from a pathogenetic or a salutogenetic perspective: 'freedom from disease or feeling of well-being', and being autonomous and able to work. Economic hardship due to expenses for medications and food for DM affected health. Individual factors such as diet, exercise and compliance with advice, and social factors with good social relations and avoidance of stress, often caused by having experienced severe events related to migrational experiences, were considered important for maintaining health and could cause DM. Disturbed relations to others (social factors), punishment by God or Fate (supernatural factors), intake of diuretics and imbalance between warmth and cold (natural factors) were also perceived as causes. A mix of biomedical and traditional explanations and active self-care behaviour with frequent use of herbs was found. It is important to assess the individual's beliefs, and health professionals, particularly nurses, should incorporate discussions of alternative treatments and other components of explanatory models and co-operate with social workers to consider influence of finances and migrational experiences on health.
\end{abstract}

Keywords: Beliefs about health/illness, Care-seeking behaviour, Diabetes mellitus, Latin Americans, migrants, self-care.

\section{INTRODUCTION}

Due to extensive global migration many European societies have become multicultural [1]. Migrants have an increased risk of developing type 2 diabetes in the emerging diabetes pandemic. The chance of encountering persons from foreign countries with different beliefs about health and illness is great in health care. Beliefs about health and illness are culturally determined and important as they guide healthrelated behaviour, including self-care and care seeking, and thus influence health [2-6]. As regards diabetes mellitus (DM), health-related behaviour is crucial for disease control and prevention of complications [7]. In nursing it is important to assess health and illness beliefs to be acquainted with the patients' self-care practices, which otherwise might counteract advice about self-care management, particularly regarding the use of medications, herbal drugs, and natural cures [8].

In Sweden about every fifth inhabitant is of foreign background (second-generation immigrants included) and more than 120 nationalities are represented [9]. One of the main non-European immigrant groups in Sweden is Latin Americans. Most immigrated during the 1970s and are refugees who fled from torture and persecution for political reasons [10]. No previous studies have been found on the

*Address correspondence to this author at the School of Health and Caring Sciences, Linnaeus University, Växjö, S- 35195 Växjö, Sweden;

Tel: +46 4707083 05; Fax: +46 470363 10; E-mail: katarina.hjelm@Inu.se topic of this study, individual beliefs about health and illness in Latin American migrants with diabetes mellitus (DM) and their influence on self-care and health care seeking. Illness perceptions, in terms of explanatory models of type $2 \mathrm{DM}$, have previously been investigated in Mexican Americans living in the USA [11, 12]. Both folk and biomedical perspectives were stated as the primary causes of DM, and expressed as susto (fright or scare), eating too many sweets, or heredity. Treatment of DM included both herbal and biomedical modalities. Many women claimed they had not used traditional remedies but that it might be helpful [11]. However, the migrational background of the informants was not described or discussed.

In studies of different migrant groups diagnosed with DM and living in Sweden, dissimilarities in beliefs were found, affecting health-related behaviour and care seeking. Middle Easterners mentioned information-seeking behaviour and thus had a low threshold for seeking care, while ExYugoslavians displayed passive self-care behaviour with low inclination to self-monitoring of blood glucose and preventive foot care, and often relied on health care staff for help, in contrast to Swedes who demonstrated an active selfcare behaviour and a healthy lifestyle [2-4]. Migrants often had a fatalistic view of the disease, and many related the causes of DM to factors outside their own control, e.g. Fate, or supernatural factors such as the will of Allah. The use of complementary alternative medicine differed; most Middle Easterners denied using it, in contrast to Ex-Yugoslavians, most of whom used different types of herbal teas as a complement to prescribed anti-diabetic drugs, while many 
Swedes used varying forms of alternative medicine, for example acupuncture, reflexology, healing, and different types of natural remedies to cure diabetes-related problems such as headache and joint pains.

The aim was to explore beliefs about health and illness in Latin American migrants diagnosed with DM and living in Sweden, and to investigate the influence on health-related behaviour including self-care and care-seeking behaviour.

\section{MATERIALS AND METHODOLOGY}

\section{Design}

Focus-group interviews were held in a qualitative exploratory study. Group interaction facilitates the respondents' ability to express and clarify their beliefs, and also encourages participants to disclose behaviour and attitudes that might not consciously be revealed in one-onone situations [13]. The technique has been considered particularly appropriate in the verbalisation of different cultural beliefs and values, and emphasises the participants' own perspective.

\section{Participants}

A purposive sampling procedure was used. Nine persons born in Latin American countries and living in Sweden participated. Criteria for inclusion were: diagnosis of DM, duration of $\mathrm{DM}>1$ year, age $\geq 20$ years, without known psychiatric disorder. All respondents had their basic management in primary health care settings, with outpatient management at health centres based on general practitioners and nurses, and were recruited by health care staff according to set inclusion criteria.

\section{Data Collection}

Data were collected through focus-group interviews. A thematised interview guide was used, with open-ended questions including descriptions of common problems related to DM. The interview guide was designed based on previous studies of persons with DM [2-4] and review of literature. Themes investigated were: content of health; factors important for health; causes, explanations and perceived consequences of diabetes; health-restorative activities; and care-seeking behaviours.

An individual standardised interview lasting for about 15 minutes, focusing on socio-demographic and medical data, was carried out before the group sessions to establish friendly contact and protect confidentiality in the group setting. The focus-groups were led by a female diabetes specialist nurse (first author) not involved in the management of the patients or in the clinic. A certified Spanish-speaking interpreter was used and the sequential interpretation technique (word for word) was applied. The interviews were held in round-table form in secluded rooms outside the clinic and respondents were grouped by gender. In order to maximise exploration of differences within the group setting, people of different ages, time of residence in Sweden, duration of DM and treatment were brought together [13]. Each focus-group comprised two to four persons, groups were held repeatedly two to four times, in free-flowing discussions lasting 1.5-2 hours, and were audiotaped and transcribed verbatim. The first session was used as a pilot test (included in the study) [13] and minor changes were made in the wording and sequencing of questions.

\section{Ethical Considerations}

The study was approved by the Lund University Ethics Committee, and was carried out with written informed consent and in accordance with the Helsinki Declaration.

\section{Data Analysis}

Collection and analysis of data proceeded simultaneously until no new information was added [13]. The analyses followed the method established for focus-groups by Krueger \& Casey [13], as previously described [2-4]. The aim was to be open to as much variation in the material as possible, searching for regularities, contradictions and patterns by comparing respondents' statements. The intention was to describe the data as closely to the material as possible and to avoid explorations on an interpretive level, which is important in studying culturally determined beliefs [14]. The lay model of illness causation [15] and the model for care-seeking behaviour [16] provided a broad theoretical framework to assist the deductive-inductive analysis [14]. In content analysis, analytical categories are often developed from different theoretical frameworks and applied to the data. Data not deductively covered by the chosen framework formed the basis for developing categories inductively from the data. Examples of the coding procedure are presented in Tables $\mathbf{2}$ and $\mathbf{3}$.

To increase the trustworthiness of the findings, the transcripts from the sessions were analysed independently by two researchers [17], a diabetes specialist nurse and a general nurse (first and second authors), and comparisons showed high agreement. Credibility is also enhanced by presenting data as categories and subcategories with illuminative quotations, and describing the audit-trail as thoroughly as possible.

\section{RESULTS}

Six women and three men, aged 36-77 years (Md 65), born in Latin American countries (Chile 7, El Salvador 1, Bolivia 1) with a median time of residence in Sweden of 17 years (range 6-30; Table 1) participated. All reported being refugees, and two had immigrated because of family ties. All except two had received their diagnosis of DM in Sweden, most were treated with oral agents, and were low educated.

\section{Beliefs About Health}

Health was described in all respondents within the category of individual factors and from four different perspectives: freedom from disease, a feeling of well-being, control/independence, and physical and intellectual health as essential for being able to work (see Table 2).

Feeling healthy was mainly ascribed to individual factors. Most discussed healthy diet and exercise and trying to feel mentally well, while some talked more generally about caring for oneself, but also a combination of individual and social factors, adding being together with the family and emphasising the importance of one's job:

I need to eat properly, ... and to exercise ... you need to eat vegetables, fruit and fish. (F7) 
To take it easy and look after yourself. (M2)

... being together with the family and my grandchildren, I think is important. (F8)

... it's work ... My job. Firstly I like it and there are always interesting things happening. (M9)

Table 1. Characteristics of the Study Population

\begin{tabular}{|c|c|}
\hline & $\begin{array}{c}\text { Latin Americans } \\
\qquad(\mathbf{N}=9)\end{array}$ \\
\hline Age $(y r)^{1}$ & $65(36-77)$ \\
\hline Female & 6 \\
\hline Male & 3 \\
\hline Time of residence in Sweden $(\mathrm{yr})^{1}$ & $17(6-30)$ \\
\hline \multicolumn{2}{|l|}{ Reason for immigration to Sweden } \\
\hline Refugee & 7 \\
\hline Refugee with family ties & 2 \\
\hline 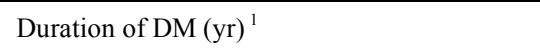 & $7(3-30)$ \\
\hline Diagnosis of DM in Sweden (n) & 7 \\
\hline \multicolumn{2}{|l|}{ Treatment of DM (n) } \\
\hline Diet & 1 \\
\hline Oral agents & 3 \\
\hline Insulin & 2 \\
\hline Combination with insulin & 3 \\
\hline \multicolumn{2}{|l|}{ Self-reported complications related to DM (n) } \\
\hline Eye & 4 \\
\hline Kidney & 1 \\
\hline Heart & 5 \\
\hline Lower extremity & 1 \\
\hline \multicolumn{2}{|l|}{ Educational level } \\
\hline$<9$ year & 4 \\
\hline Upper secondary school (9-12 yrs) & 2 \\
\hline University $<2$ yrs & 2 \\
\hline University $>2$ yrs & 1 \\
\hline Students (n) & 1 \\
\hline Work (part/full-time) (n) & 2 \\
\hline Early retirement pensioners (n) & 2 \\
\hline Old-age pensioners (n) & 4 \\
\hline \multicolumn{2}{|l|}{ Family circumstances } \\
\hline Married & 2 \\
\hline Widow & 3 \\
\hline Divorced & 4 \\
\hline
\end{tabular}

Factors of importance for health were related to individual factors. Most mainly talked about 'exercise and diet' (F3) or the 'emotional status ... something that is not in agreement with my feelings' (F6) while some said they didn't know as 'this is new to me' (M1) or 'nothing, as I can't accept this disease' (M1).

All respondents considered their finances important for health, but only two said they could cope completely with the situation. The disease caused economic hardship because of increased costs for healthy food and medications. Some spoke of being unable to collect prescribed medications from the pharmacy or having to purchase by instalments.

It's expensive ... the cost is considerable. Before...the same food for the whole family and now you have to follow a certain diet ... if you're prescribed medicine you have to go to the pharmacy and it's a big expense. (M1)

The one who is working is unable to go to the social services to say that I have now had these expenses so I need compensation... When I buy medication...I have been lucky to...buy on credit...many ...can't afford the medications. (F6)

As regards finances ... I can manage ... I bake my own bread ...I freeze it ...(F8)

Most considered instrumental tangible support (social factors) from the family or friends important for health in terms of practical help in following diet prescriptions, understanding the situation, and being able to discuss it with others. Finally, one man talked about the importance of medications (natural factors). Health care staff were seen as a source of help, support and control of DM. Some asked for informative support as they lacked information.

when I'm depressed ... sad and don't feel well ... they touch me and take my hand ... that brings peace. This human warmth ... their benevolence in listening to me ... even if we have language difficulties $\ldots$ to try to understand how I feel. (F6)

Factors considered harmful to health were mainly individual and social, such as stress and strained relations, making respondents feel depressed, angry or stressed. A few discussed influence of limited exercise and unhealthy diet with too much sugar:

Stress ... Housework and when you have... children. I get high blood pressure. (F5)

Sugar... I have stopped ... drinking alcohol ... you have to take exercise all the time, then it's easier to keep blood glucose on a lower level. (M2)

To maintain health and prevent complications related to DM, respondents talked about three main categories, two of these related to individual lifestyle, chiefly the healthy diet but also exercise and following prescribed medication, and one to social factors exemplified as good relations to others.

... this thing about diet ... Exercise, get out walking. Important to keep your weight ... the same size. (F5)

I follow the doctor's prescriptions ... not to forget medication, take it at the right time. (F3) 
Table 2. Beliefs About Health

\begin{tabular}{|c|c|c|}
\hline Main Analytical Category & Subcategory & Quotation \\
\hline \hline Individual factors & Freedom from disease & 'that you do not have any disease' (F4) \\
\hline & A feeling of well-being & 'to feel good' (F 3) \\
\hline & 'to have control over oneself' (M1) \\
\hline & $\begin{array}{c}\text { Physical and intellectual health as a } \\
\text { prerequisite for being able to work }\end{array}$ & $\begin{array}{c}\text { 'Health allows me to work ... work and health are related ... a physical health } \\
\text { status ... and a healthy mind so that one can function intellectually' (M9) }\end{array}$ \\
\hline
\end{tabular}

... a harmony ... that mostly has to do with human relations ... it's when we're near each other ... the close relation that is important. [Interviewer: What happens if you don't have a close relationship?] A lot of stress will arise, and sleep will be disturbed ... blood counts will change ... poor digestion ... constipation. (M9)

To improve their health most of the respondents used natural remedies, such as various herbal teas, often bought in their home countries. Complementary alternative medicine measures were used to a limited extent; one person had turned to a traditional healer to get herbs to treat kidney problems, another had used acupuncture against pain, and two others had either used exercise in water or self-massage of the feet and drinking a lot of water:

I drink some decoctions of different herbs ... I drink it as water before breakfast... at lunch and in the evening. These products are homeopathic. I order the tea from Chile. (M1)

I had problems with my kidneys in Chile and I didn't visit a doctor for a long time. Then I got help from a female traditional healer ... using natural medications ... based on herbs. (M2)

Religion was considered important for health by all except one, and the majority were Catholics, who claimed it helped them to change things; several said it helped them to 'move mountains'.

For all, except two men, it was very important to celebrate traditional feasts, particularly to be able to meet the family, relatives and friends:

....all gather, different generations...family, children, grandchildren and other ... friends ...

For example when we celebrate the national day ... We meet... and make traditional food and ... have music and dance. (F8)

Some also said they had adapted their customs to Swedish ones, e.g. Christmas food.

Only one person had participated in a self-help group for diabetic persons but had stopped as it was considered of "no use'. Most knew about them and would like to participate but found language barriers a hindrance:

\section{Beliefs About Illness}

All respondents except two had been diagnosed in Sweden. One person had been diagnosed and self-treated for
DM by his grandmother (popular sector) in the home country, as several in his family had the disease. He was the only who then had consulted a physician (professional sector) in Sweden as he knew his symptoms were caused by DM and needed treatment:

being thirsty ... had to urinate several times a night ... problems with the eyes ... in my family diabetes has also been evident ... grandmother said we had diabetes ... she was our physician ... she made a special diet ... based on fish and vegetables ... when I came to Sweden I took a test ... was told I had diabetes ... first a test with tablets and then injections. (M9)

The other respondents had not suspected DM and most had been diagnosed by coincidence as a secondary finding to other diseases when consulting a doctor or being admitted to hospital for e.g. coronary heart infarction, pneumonia, kidney problems, or having consulted a physician mainly in a health centre (professional sector) for symptoms such as dizziness, nausea, headache, and/or numbness in the lower extremities.

I had no symptoms ...I came to the physician with pneumonia. In this examination ... it was detected that I had diabetes (F8).

The reactions to the diagnosis varied from a crisis where 'the whole world fell down ... I cried a lot' (F8), to 'acceptance and resignation' (F3), to 'I did not react in any particular way ... as I didn't know how dangerous this disease is or what it can lead to' (F5), and finally 'being calm as whole my family has had diabetes' (M9).

The causes of DM varied; some had been informed by the physician 'that it was this infection' or 'the kidneys' (individual and social factors, F8, F7), others said 'I can't find any cause' (F5) or discussed intake of too much sugar or heredity (individual factors) (Table 3). Also the influence of severe migrational experiences in the home country on the body (social and individual factors) and changed preparations of food affecting the pancreatic function (natural factors) were discussed. When a list of potential causes of DM was discussed, most talked about individual factors, such as unhealthy diet, obesity, and inactivity, and social factors like stress. Social factors such as disturbed relations to others (alive or dead), supernatural factors such as punishment by God or Fate, and natural factors such as intake of diuretics, and imbalance in the body between warmth and cold or yin and yang were also added. 
Table 3. Beliefs About Causes of Diabetes Mellitus

\begin{tabular}{|c|c|c|}
\hline $\begin{array}{l}\text { Main Analytical Category }{ }^{1} \\
\text { Number of Statements (n) }\end{array}$ & Subcategory & Quotation \\
\hline \multicolumn{3}{|c|}{ Factors Related to the Individual (38) } \\
\hline & Intake of sugar & $\begin{array}{l}\text { 'I didn't know exactly, thought that it was because one } \\
\text { used sugar or ... ate too much chocolate or sweets ... I } \\
\text { used to drink coffee ... with three spoons of sugar' }\end{array}$ \\
\hline & Heredity & $\begin{array}{l}\text { 'has to do with the family, my mother had diabetes and } \\
\text { my sisters and brothers ...' }\end{array}$ \\
\hline & Heredity. $^{2}$ & \\
\hline & Unhealthy dietary habits. $^{2}$ & \\
\hline & Obesity. $^{2}$ & \\
\hline & Pancreatic diseases. ${ }^{2}$ & \\
\hline & Inactivity. $^{2}$ & \\
\hline & Infections. $^{2}$ & \\
\hline & Pregnancy. ${ }^{2}$ & \\
\hline & Imbalance between warmth/cold or yin/yang. ${ }^{2}$ & \\
\hline \multicolumn{3}{|l|}{ Factors Related to Nature (3) } \\
\hline & Decreased pancreatic function due to intake of margarine. & $\begin{array}{l}\text { 'as the pancreas works less well and the production of } \\
\text { insulin decreases. Since I came to Sweden } 25 \text { years ago } \\
\text { and started to cook with margarine ... it's not good'. }\end{array}$ \\
\hline & Intake of diuretics. $^{2}$ & \\
\hline \multicolumn{3}{|c|}{ Factors Related to the Social Sphere (12) } \\
\hline & Stress $^{2}$ & \\
\hline & Disturbed relations to others, alive or dead. ${ }^{2}$ & \\
\hline \multicolumn{3}{|c|}{ Factors Related to the Individual and Social Sphere (3) } \\
\hline & The physician has informed about the cause & '... they said - the physician - it was this infection' \\
\hline & Experiences in the home country causing mental trauma & $\begin{array}{l}\text { '... what I experienced in my home country. Such shocks } \\
\text { where horror is involved ... can cause big changes in the } \\
\text { organism ... mental illness, depressions ...' }\end{array}$ \\
\hline \multicolumn{3}{|c|}{ Factors Related to the Supernatural Sphere (4) } \\
\hline & Fate $^{2}$ & \\
\hline & Punishment from God ${ }^{2}$ & \\
\hline \multicolumn{3}{|l|}{ Don't Know (1) } \\
\hline & No cause $^{1}$ & I can't find any cause \\
\hline
\end{tabular}

'Analytical categories according to the lay model of illness causation by Helman (2007) [15], in which causes of illness could be related to the individual, nature, social relations and/or the supernatural world.

${ }^{2}$ Findings from discussions of a list of potential causes of diabetes mellitus.

In general, limited knowledge was demonstrated when discussing what happens in the body when one has DM, the effects of insulin, the pancreatic function and the action of antidiabetic drugs. However, knowledge about medications was better in those treated with insulin. Most named DM 'sugar disease' and knew that it was related to 'lack of insulin'. Only one person said that medications 'influence the level of blood glucose' and another that 'if one does not manage the diabetes there is a series of complications, kidneys, heart, eyes ...' (F5). Most perceived DM to be lifelong, while one claimed it was related to chance and another hoped for the development of a cure. The respondents did not express any future fears of the disease, with two exceptions who discussed worries about complications such as amputations, blindness, and disablement.

Participants stated that lifestyle changes was the problems they experienced following the disease:

...the change we had to go through. As with the diet, for example, change all dietary habits ... Give up all the good things ... Meals have become more regular, breakfast, lunch ...(F8) 
Others discussed medical problems such as poor blood circulation, loss of memory and shortness of breath during the night, and endocrinological problems with changed temper, being more aggressive.

\section{Self-Care and Care-Seeking Pattern}

Respondents spoke about being informed about regular check-ups, mostly 'twice a year' which they claimed to follow. Advice about foot care varied. Some said 'you need to check your feet' (M2), others had been informed about 'caring for the feet but not how' (F5) and some said they had not received any information. Thus, limited information was demonstrated.

Most had been informed about self-monitoring of blood glucose but the frequency varied, and some said that blood glucose was only checked by health professionals. However, only three persons made regular controls. Some claimed they could learn how their blood glucose was by 'measuring (blood glucose) myself many times a day ... I feel calm when I see how the glucose is. So I really have control of these figures' (M9), while others relied on health care staff, 'following these controls by the district nurse' (F6).

When discussing dietary recommendations the content varied but most had been informed about reduction of fat and increased intake of vegetables rich in fibres. Only one claimed to have been informed about the importance of regular meals. All claimed to follow advice received, although some admitted it was sometimes problematic.

...advised to eat more vegetables...And white meat, chicken, fish and turkey ... I have another enemy ... I am obese... have problems getting rid of it. (M1)

All had been told about the importance of exercises, such as 'walking a lot' and claimed 'I follow all advice' (F8). This was also evident concerning intake of medications and 'always at the same time, half an hour before the meal' (F5). There was one exception, a woman who said:

I haven't eaten them (antidiabetic drugs) for 3 months. But I eat pills, I'm going to buy them again now ... I haven't had enough money to buy them. It's so expensive. (F4)

Common health problems in DM included: impaired glycaemic control, hyperglycaemia, repeated episodes of hypoglycaemia, gastrointestinal infection, common cold and pharyngitis, urinary infection, foot problems (crawling, burning, decreased sensitivity, wounds), spasm in the calf, hypertension and albuminuria. A pattern was evident here, as most respondents identified the causes of the problems, used self-care measures (individual factors), and sought help from health care staff (physicians and nurses) in the professional sector if needed. In case of a common cold and pharyngitis or gastrointestinal infection, respondents described self-care measures such as home remedies, mainly using herbs, e.g. mint, camomile (natural factors), to make tea. Drinking warm beer with salt was also tried.

Now when I have a slight cold ... I make lemon water ... in the evening...milk with some kind of herb...(F6)
There's an infection in my stomach. I don't take any medication, instead I drink green tea with...lemon and honey $\ldots$ and rest. [Interviewer: Have you ever sought help for this?] Yes, the family doctor. He said it was normal. (M9)

The contact with health care staff managing their DM was perceived as unproblematic, except that some wanted more follow-ups, more written information and in their own language (Spanish). Suggestions for improvement included ability to communicate with health care staff in their mother tongue.

...no particular problems ... the care is good but we lack ... written information. With $\mathrm{X}$ (Spanish-speaking nurse) it is easier ... if you don't manage yourself she tells you sharply ... when you go to buy food there's not much that suits diabetics. (M2)

\section{DISCUSSION}

This study is unique as it explores individual beliefs about health and illness in Latin American migrants and their influence on self-care and care-seeking behaviour. The main findings showed that health was viewed from a pathogenetic or a salutogenetic perspective (freedom from disease or feeling of well-being), being autonomous and able to work. Limited budget related to the cost of diabetes medications and food affected health. Individual factors such as diet, exercise and compliance with advice, and social factors with good social relations and avoidance of stress, were considered important for health and major factors causing DM. Natural remedies, particularly different herbs, were frequently used in active self-care, and help from health professionals was sought to a limited extent.

In this study beliefs about health were expressed both from a pathogenic and a salutogenetic point of view [18], and from an action-theory point of view [19] illustrated by the ability to be independent/autonomous, controlling the situation and being able to work. Health is then defined as being able to act in accordance with one's own plan or goal in life under the prevailing environmental restrictions [19]. Previous studies of migrants with DM living in Sweden have shown the importance of having a job and being active for experiencing good health [3, 4]. Unemployment and experiencing immigrant status are common in non-European immigrants, mainly refugees [20], and contribute to perceived stress leading to poor glycaemic control and bad mood, which might cause conflicts within the family and start a vicious circle negatively affecting the disease [4]. This needs to be recognised and prevented in order to promote health in diabetic subjects.

When studying beliefs about health, individual and social factors expressed as a healthy lifestyle (appropriate diet, exercise, compliance with advice) and good social relations and avoidance of the stress often related to severe migrational experiences, were considered important to promote and maintain health. In comparison to results from other migrant groups previously studied in Sweden, ExYugoslavians and Middle Easterners [3, 4], this pattern was repeated throughout the interviews. The focus on relations to 
others is possibly explained by the fact that all respondents reported being refugees and had experienced different types of persecution, torture, loss of home, and other severe events related to migration. Latin American refugees have reported high degrees of psychological distress, possibly explained by experiences before and during the migration, but also the great geographical and cultural distance in perceived relations with Swedes [21]. Alienation, social degradation and social isolation have been described by Latin American refugees living in Sweden. It could also be related to the origin of the respondents in cultures described as collectivistic [22], where the role of kin and extended family members and thus, relations to others, is well documented $[23,24]$. Immigrants have to deal not only with the chronic disease but also broken or weak social networks [25, 26], lack of social support, social isolation and alienation in a foreign culture [27].

The respondents in this study showed active self-care behaviour with additional limited help sought from health professionals and others in the professional $v s$ popular sector. Frequent use of natural remedies, particularly herbs, was said to improve and restore health. Thus, their healthrelated behaviour was similar to the active self-care behaviour and healthy lifestyle found in Swedes with DM, in contrast to migrants from the Middle East, who seek information and have a low threshold for seeking care, and Ex-Yugoslavians who rely on health care staff for help and have a passive attitude to self-care $[3,4]$. On the other hand, they showed similarities to Ex-Yugoslavians who also frequently used herbal remedies as a complement to prescribed medication. Previous studies of Mexican Americans [12, 28] and Nicaraguans [29] have shown frequent use of herbal remedies for a variety of symptoms, often along with or instead of prescribed medications. Countries in Latin America have a deeply ingrained history of using herbal remedies. Home and herbal remedies may be significantly cheaper and more easily available to patients than allopathic medication [28, 29]. In the present study respondents indicated the economic factors as important for health, since medications and food for DM are costly. Need for economic support has previously been claimed in foreign-born persons with DM living in Sweden [30] as the costs of healthy food (e.g. vegetables, bread rich in fibre, low in fat) are higher than for other types of food. Many foreignborn persons in Sweden, particularly non-Europeans, live in strained economic circumstances due to dependency on social allowance, low pensions, and unemployment [20] which needs to be considered in diabetes care. Cooperation with social workers should be undertaken to assist with adequate support [30]. Further, it is of the utmost importance to assess patients' use of herbal remedies, particularly if used as an alternative to allopathic medicine [28,29], to avoid a switch between different caregivers that might interrupt compliance and glycaemic control, negatively affecting health [31]. Nurses need to elicit information about use from patients in a sensitive and non-judgemental manner, as many users believe that health care providers would admonish them for it and thus do not inform about the treatments used [28]. It is also important to check whether there could be any kind of interactions that need to be prevented.
Although the results indicated limited knowledge about $\mathrm{DM}$, insulin and pancreatic function, knowledge of the causes of DM varied but was adequate. A combination of individual factors, e.g. unhealthy diet, obesity and pancreatic diseases, and social factors such as stress and experiences of severe events related to migrational experiences were mainly stated. Thus, this migrant group differs in beliefs about illness from the previously studied Ex-Yugoslavians and Middle Easterners, indicating limited knowledge and mainly focusing on supernatural factors such as the influence of Fate and the will of Allah, but is similar in also focusing on the stress caused by migration [2-4]. The findings from the open-ended questions were rather limited in content and focused on individual and social factors (pancreatic function, diet, severe events related to migration) and some were unsure of the cause. When a list of potential causes was additionally discussed, the pattern persisted but the content was extended with other social factors such as stress and relations to others, influence of supernatural forces (Fate, the will of God) and natural factors (treatment with diuretics). Thus, the whole spectrum of main causes described by the lay theory model of illness causation by Helman [15] was illustrated and, as in previous studies of Mexican Americans living in the USA [11, 12], both folk and biomedical perspectives were held. The previously described cause of diabetes expressed as susto - fright or scare [11,12] - might be similar to the experiences described of severe events related to migration but expressed in another way. The assumptions made in the lay theory model of illness causation that non-westerners mainly tend to focus on supernatural and social factors and thus have a fatalistic view of the disease and an external locus of control [32], are not supported by the findings in this study. The active self-care pattern in addition demonstrates the contrary, and an internal locus of control [32] is essential for good management of DM. As previously concluded [3,4], it is important to assess the individual's own beliefs and avoid crude generalisations among persons of different origin. Nurses should incorporate in their practice a discussion of alternative treatments as well as other components of the patients' explanatory models [33].

It is noticeable that the respondents in general claim to follow advice for self-care but to have received limited advice about foot care and regular meals. It may be wondered whether they have received advice or limited advice or not understood the advice given. In any case, there was a call for information and communication in their own language which needs to be supported, as previously demonstrated in studies of foreign-born diabetic persons' own perceptions of the content of and desire for support [30].

The number of participants $(\mathrm{N}=9)$ is limited but the analysis of data showed that saturation was reached [13]. The sample included more women than men $(\mathrm{n}=6$ vs 3$)$, which reflects how prevalence of DM has increased in women of reproductive age, and due to differences in lifeexpectancy there are more women than men with diabetes in older age groups [7]. Gender is not problematised in this study, as it is a first explorative step, which is a limitation as dissimilarities might be revealed and need to be further 
investigated. Results of qualitative studies might be seen as limited concerning the possibility to generalise findings [17]. In this study the aim was to disclose different beliefs in a group of persons with DM and to find unique understandings instead of data generalisable to the whole population. Qualitative data are transferable to other similar groups if carefully collected and analysed [17]. It is important to consider that the study population included persons identifying themselves as refugees and that persons with other types of migrational background might differ. However, the findings give perspectives that are important to elicit in assessing beliefs to plan for individualised care.

\section{CONCLUSION}

Both biomedical and traditional explanations were found, and active self-care behaviour with frequent use of herbal remedies. Economic factors were considered important for health, and costly diabetes diet and medications had a negative influence. It is important to assess the individual's own beliefs, and health professionals, particularly nurses, should incorporate in their practice a discussion of alternative treatments and other components of the patients' explanatory models and cooperate with social workers to consider the influence of finances and migrational experiences on health.

\section{CONFLICT OF INTEREST}

The authors confirm that this article content has no conflict of interest.

\section{AUTHOR CONTRIBUTIONS}

Study design: $\mathrm{KH}$

Data collection and analysis: $\mathrm{KH}, \mathrm{KB}$

Manuscript preparation: $\mathrm{KH}, \mathrm{KB}$

\section{ACKNOWLEDGEMENTS}

This work was supported by grants from the Faculty of Medicine, Lund University, the Swedish Foundation for Health Care Sciences and Allergy Research (Vårdalstiftelsen), the Swedish Diabetes Association (Svenska Diabetesförbundet), and the research profile AMER (Arbetsmarknad, Migration, och Etniska Relationer; Labour Market, Migration and Ethnic Relations), Växjö University

\section{REFERENCES}

[1] Irving G, Mosca D. Future capacity needs in managing the health Aspects of migration. International Organization For Migration (IOM), 2010. [cited: 2012 August 15] Available from: http://publi cations.iom.int/bookstore/free/WMR2010_capacity_nee ds_health_aspects.pdf

[2] Hjelm K, Nyberg P, Isacsson Å, Apelqvist J. Beliefs about health and illness essential for self-care practice: a comparison of migrant Yugoslavian and Swedish diabetic females. J Adv Nurs 1999; 30: $1147-59$

[3] Hjelm K, Bard K, Nyberg P, Apelqvist J. Religious and cultural distance in beliefs about health and illness in diabetic women of different origin living in Sweden. Int J Nurs Stud 2003; 40:627-43.

[4] Hjelm K, Bard K, Nyberg P, Apelqvist J. Beliefs about health and diabetes in men with diabetes mellitus of different origin living in Sweden. J Adv Nurs 2005; 50: 47-59.
[5] Hjelm K, Mufunda E. Zimbabwean diabetics' beliefs about health and illness: an interview study. BMC Int Health Hum Rights 2010; 10: 7 .

[6] Glanz K, Rimer B, Lewis F. Health behaviour and health Education: theory, research, and practice. $3^{\text {rd }}$ ed. San Francisco: JosseyBass 2002.

[7] International Diabetes Federation. Diabetes Atlas. $4^{\text {th }}$ ed. 2009 [cited: 2012 August 15]. Available from: www.diabetesatlas.org.

[8] Leininger MM, McFarland MR. Culture care diversity and Universality: A worldwide nursing theory. $2^{\text {nd }}$ ed. Boston: Jones and Bartlett 2006.

[9] SOS (2011) Sveriges Officiella Statistik. Befolkningsstatistik. (Official Statistics of Sweden. Population Statistics) 20111231.

[10] Svanberg I, Tydén M. Tusen år av invandring: En svensk kulturhistoria. (A thousand years of immigration: A Swedish cultural history). Stockholm: Gidlunds bokförlag 1992.

[11] Alcozer F. Secondary analysis of perceptions and meanings of type 2 diabetes among Mexican American women. Diabetes Educ 2000; 26:785-95.

[12] Jezewski MA, Poss J. Mexican Americans' explanatory model of type 2 diabetes. West J Nurs Res 2002; 24:840-58.

[13] Krueger RA, Casey MA. Focus groups: A practical guide for applied research. $4^{\text {th }}$ ed. London: Sage Publications 2009.

[14] Krippendorff K. Content analysis: An introduction to its methodology. London: Sage Publications 2004.

[15] Helman C. Culture, health and illness. London: Butterworth \& Co 2007.

[16] Kleinman A. Patients and healers in the context of culture. London: University of California Press, Ltd 1980.

[17] Patton MQ. Qualitative research \& evaluation methods. $3^{\text {rd }}$ ed. London: Sage 2004.

[18] Antonovsky A. Unraveling the mystery of health. San Francisco: Jossey-Bass Inc. 1987.

[19] Pörn I. Health and adaptedness. Theor Med Bioeth 1993; 14: $295-$ 303.

[20] Socialstyrelsen. Folkhälsorapport.2009 (The National Board of Health and Welfare. Public Health Report). [cited: 2012 August 15]. Available from: http://www.socialstyrelsen.se/Lists/Artikelkat alog/Attachments/849 5/2009-126-71_200912671.pdf

[21] Sundquist J. Refugees, labour migrants and psychological distress. A population-based study of 338 Latin-American refugees, 161 south European and 396 Finnish labour migrants, and 996 Swedish age-, sex- and education-matched controls. Soc Psychiatry Psychiatr Epidemiol 1994; 29: 20-4

[22] Hofstede G. Culture's consequences: International differences in work-related values. London: Sage Publications 1984.

[23] Hurtado A. Variations, combinations, and evolutions: latino families in the United States. In: Zambrana RE, Ed. Understanding Latino families: Scholarship, policy and practice. London: Sage Publications Inc. 1995.

[24] Pernice-Duca FM. An examination of family and social support networks as a function of ethnicity and gender: A descriptive study of youths from three ethnic reference groups. J Youth Stud 2010; 13:391-402.

[25] Hull H. Migration, adaptation and illness: a review. Soc Sci Med 1979; 13: 25-36.

[26] Kirkcaldy K, Wittig U, Furnham A, Merbach M, Siefen RG. Migration und Gesundheit: Psychozociale Determinanten. Bundesgesundheitsblatt Gesundheitsforschung Gesundheitsschutz 2006; 49: 873-83

[27] Andersson J. Immigrant women speak of chronic illness: the social construction of the devalued self. J Adv Nurs 1991; 16: 710- 17.

[28] Poss E, Jezewski MA, Stuart AG. Home remedies for type 2 diabetes used by Mexican Americans in El Paso, Texas. Clin Nurs Res 2003; 12: 304-21.

[29] Ailinger R, Molloy S, Zamora L, Benavides C. Herbal remedies in a Nicaraguan Barrio. J Transcult Nurs 2010; 15: 278-82.

[30] Berterö C, Hjelm K. Social support as described by foreign-born persons diagnosed with type 2 diabetes mellitus and living in Sweden. Nurs Health Sci 2010; 12: 507-14.

[31] Srinivas G, Suresh E, Jagadeesan M, Amalraj E, Datta M. Treatment-seeking behaviour and compliance of diabetic patients in a rural area of South India. Ann NY Acad Sci 2002; 958: 420- 4. 
[32] Rotter JB. Generalized expectancies for internal versus external control of reinforcement. Psychol Monogr 1966; 80(1): 1-28.
[33] Hatcher E, Whittemore R. Hispanic adults' beliefs about type 2 diabetes: Clinical implications. J Am Acad Nurs Pract 2007; 19: $536-45$.

(C) Hjelm and Bard; Licensee Bentham Open.

This is an open access article licensed under the terms of the Creative Commons Attribution Non-Commercial License (http://creativecommons.org/licenses/by-nc/3.0/) which permits unrestricted, non-commercial use, distribution and reproduction in any medium, provided the work is properly cited. 